\title{
Ultrasound biomicroscopy of Chinese eyes with iridocorneal endothelial syndrome
}

\author{
M Zhang, J Chen, L Liang, A M Laties, Z Liu
}

Br J Ophthalmol 2006;90:64-69. doi: 10.1136/bjo.2005.074864

See end of article for authors' affiliations .....................

Correspondence to: Zuguo Liv, MD, PhD, Zhongshan Ophthalmic Center and Ocular Surface Center, 54 S Xianlie Road, Guangzhou 510060, PR China: zuguol@yahoo.com

Accepted for publication 2 September 2005

\begin{abstract}
Aim: To document the ultrasound biomicroscopic (UBM) findings in Chinese patients with iridocorneal endothelial (ICE) syndrome.

Methods: 21 patients with ICE syndrome and 15 normal subjects underwent UBM. UBM findings of anterior segment were compared between normal subjects and three clinical types of ICE syndrome: progressive iris atrophy (PIA), Chandler's syndrome (CS), and Cogan-Reese syndrome (CRS).

Results: Central anterior chamber depth was significantly less in patients with ICE syndrome (2.25 (SD $0.32) \mathrm{mm}$ ) than in normal subjects $(2.76(0.32) \mathrm{mm})$. Peripheral anterior synechiae were observed in all the ICE patients by UBM. Three out of four CRS subjects showed an "arborised" shape of iridocorneal angle. Two eyes out of 10 with CS presented bridge-shaped synechiae. A membrane-like mound was observed in iridocorneal angle in two patients: one with CRS and one with CS. UBM was found to be more effective in detecting peripheral anterior synechiae (PAS) and iris atrophy than slit lamp microscopy and gonioscopy, mainly because of corneal oedema in patients with CS. Four out of 11 patients with unilateral ICE syndrome had shallow or closed anterior chamber angles in their fellow eyes. Two of them successfully responded to laser peripheral iridotomy.

Conclusions: UBM is an effective method to reveal the anterior segment features and provides a useful tool in the diagnosis of ICE syndrome. Different subtypes of ICE syndrome may have different UBM manifestations. UBM can help to identify angle closure in the fellow eye of unilateral ICE syndromes.
\end{abstract}

I idocorneal endothelial (ICE) syndrome is a rare disease characterised by corneal endothelial abnormality, peripheral anterior synechiae (PAS), iris stromal abnormalities, and secondary glaucoma. ICE syndrome can be subdivided into progressive iris atrophy (PIA), Chandler's syndrome (CS), and Cogan-Reese syndrome (CRS). ${ }^{1}$ Without proper treatment, blindness follows corneal oedema and secondary glaucoma in many cases..$^{2-5}$ The prevalence of glaucoma associated with ICE syndrome ranges from $46 \%$ to $82 \% .^{6-9}$ In most cases, the glaucoma is refractory, requiring multiple medications and surgical procedures..$^{3-5}$ Although patients with ICE syndrome have distinctive clinical features, diagnosis proves difficult in some cases. ${ }^{910}$ For instance, Laganowski et al reported that $68 \%$ of the ICE patients with glaucoma were overlooked initially. ${ }^{9}$ Lack of awareness of this relatively rare disorder is one explanation for misdiagnosis. Another may be that diagnosis is hindered by corneal oedema obscuring the anterior chamber angle.

Ultrasound biomicroscopy (UBM) has a significant role in ocular imaging in recent years. It has allowed progress in the study of glaucoma and has contributed to a better understanding of the mechanisms of angle closure in glaucoma. ${ }^{11}{ }^{12}$ In addition, UBM has been used to study structural alterations in many other pathological conditions such as aniridia, ocular trauma, and so on. ${ }^{13-15}$ Also, UBM has proved to be useful for evaluating the status of surgical procedures such as laser iridotomy. ${ }^{16}$ In particular, it has permitted visualisation of the anterior chamber where gonioscopy is not feasible. ${ }^{17}{ }^{18}$ Surprisingly, no UBM study on ICE syndrome has been reported in spite of the high prevalence of glaucoma and corneal oedema in these patients. The aim of the present study was to use UBM to characterise the morphological features in eyes with ICE syndrome of different types.

\section{PATIENTS AND METHODS}

\section{Patients}

The records of the patients diagnosed with ICE syndrome and seen at both the glaucoma department and the cornea department in the Zhongshan Ophthalmic Center, Sun Yatsen University between January 1998 and February 2003 were reviewed retrospectively. Twenty one patients with ICE syndrome who underwent UBM before any surgical procedure were included in this study. Patients with a history of ocular trauma or uveitis were excluded. The diagnosis and the classification of ICE syndrome of different subtypes were determined by the examining physician. Inclusion criteria were "hammered silver" appearance of posterior corneal surface or corneal oedema that precluded visualisation of the posterior cornea, and characteristic iris stromal abnormalities including PAS, corectopia, iris holes, or multiple iris nodules. Patients were classified into three clinical variants based on iris changes. ${ }^{7}$ Progressive iris atrophy was diagnosed in patients with iris stromal atrophy and distortion with or without iris holes. Those with multiple iris nodules were classified as CRS. CS was indicated by the presence of corneal abnormalities typical of ICE but with minimal or no iris stromal atrophy.

Among 21 patients, 10 patients (11 eyes) were examined for the affected eye and seven patients (seven eyes) were examined for both affected and the fellow eyes. The remaining four patients (four eyes) had surgery before receiving UBM examination. Their fellow eyes received UBM examination during follow up, so only the UBM records for the fellow eyes for these four patients were

\footnotetext{
Abbreviations: $A C D$, anterior chamber depth; BCVA, best corrected visual acuity; CS, Chandler's syndrome; CRS, Cogan-Reese syndrome; ICE syndrome, iridocorneal endothelial syndrome; PACG, primary angle closure glaucoma; PAS, peripheral anterior synechiae; PIA, progressive iris atrophy; UBM, ultrasound biomicroscopy
} 


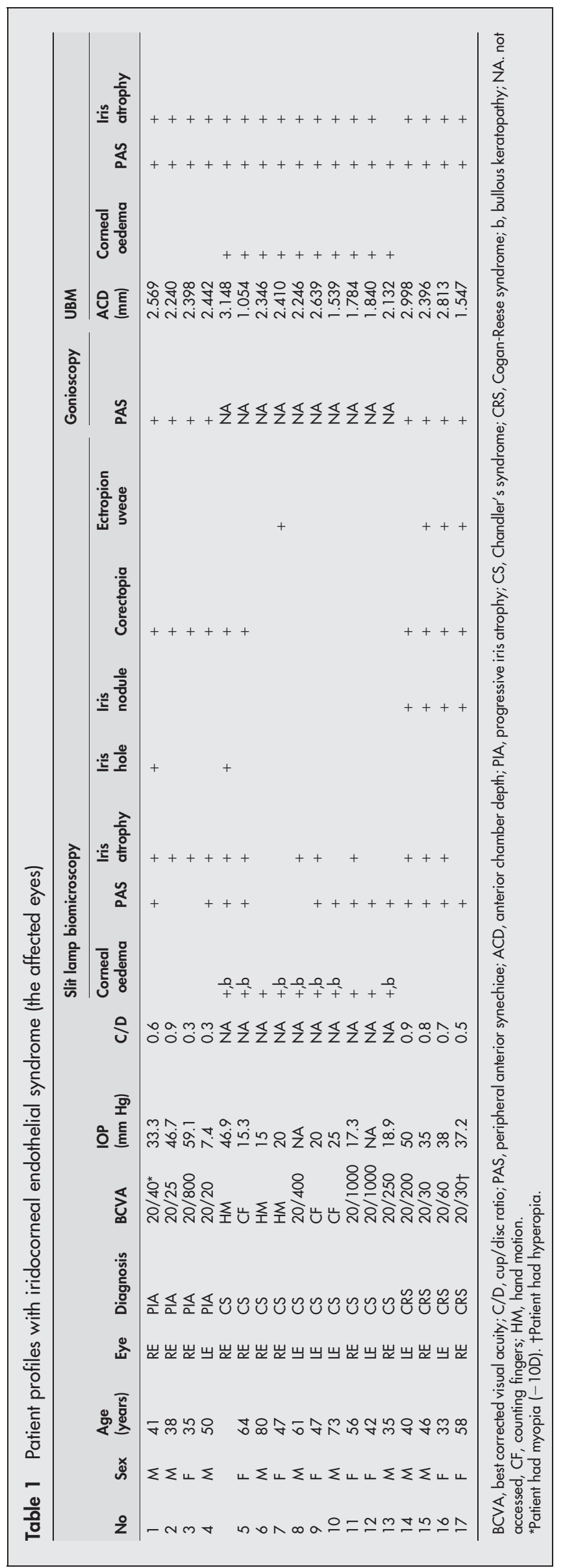


Table 2 Patient profiles with iridocorneal endothelial syndrome (the fellow eyes)

\begin{tabular}{|c|c|c|c|c|c|c|c|c|c|c|c|c|}
\hline \multirow[b]{2}{*}{ No } & \multirow[b]{2}{*}{ Sex } & \multirow[b]{2}{*}{$\begin{array}{l}\text { Age } \\
\text { (years) }\end{array}$} & \multirow[b]{2}{*}{ Eye } & \multirow[b]{2}{*}{$\begin{array}{l}\text { Diagnosis } \\
\text { (affected } \\
\text { eye) }\end{array}$} & \multirow[b]{2}{*}{ BCVA } & \multirow[b]{2}{*}{$\begin{array}{l}\text { IOP } \\
(\mathrm{mm} \mathrm{Hg})\end{array}$} & \multirow[b]{2}{*}{$C / D$} & \multicolumn{5}{|l|}{ UBM } \\
\hline & & & & & & & & $\begin{array}{l}\text { ACD } \\
(\mathbf{m m})\end{array}$ & $\begin{array}{l}\text { Iris } \\
\text { bombé }\end{array}$ & $\begin{array}{c}\text { Narrow } \\
\text { angle }\end{array}$ & PAS & $\begin{array}{l}\text { Ciliary } \\
\text { body } \\
\text { cyst }\end{array}$ \\
\hline 2 & $M$ & 38 & LE & PIA & $20 / 20$ & 15 & 0.4 & 2.95 & & & & \\
\hline 7 & $\mathrm{~F}$ & 47 & LE & CS & $20 / 20$ & 14 & 0.3 & 2.385 & & & & \\
\hline 8 & M & 61 & RE & CS & $20 / 30^{*}$ & 10.2 & 0.4 & 2.75 & & & & \\
\hline 11 & $\mathrm{~F}$ & 56 & LE & CS & $20 / 20$ & 15.9 & 0.3 & 2.06 & & & & \\
\hline 14 & $M$ & 40 & RE & CRS & $20 / 20$ & 14 & 0.4 & 2.23 & & & & + \\
\hline 15 & $M$ & 46 & LE & CRS & $20 / 20$ & 16 & 0.5 & 3.5 & & & & \\
\hline 16 & $\mathrm{~F}$ & 33 & RE & CRS & $20 / 20$ & 18 & 0.4 & 2.79 & & & & \\
\hline 18 & $\mathrm{~F}$ & 34 & RE & CS & $20 / 20$ & 17.3 & 0.3 & 1.84 & + & + & + & \\
\hline 19 & $\mathrm{~F}$ & 51 & RE & $\mathrm{PIA}, \mathrm{CS}$ & $20 / 20 \dagger$ & 17.3 & 0.3 & 1.644 & + & + & & \\
\hline 20 & $\mathrm{~F}$ & 55 & RE & PIA & $20 / 20$ & 13.7 & 0.4 & 1.852 & + & + & & \\
\hline 21 & $M$ & 50 & RE & CS & $20 / 30$ & 15.2 & 0.4 & 1.887 & + & + & & \\
\hline
\end{tabular}

All data come from the fellow eyes except the diagnosis. Patients $2,7,8,11,14,15$, and 16 received UBM in both eyes. *Patient had myopia. †Patient had hyperopia.

included in this study. Among 18 affected eyes that received UBM, four eyes were diagnosed as having PIA, 12 eyes as having CS, and four eyes as having CRS. The profiles of the patients are summarised in tables 1 and 2 .

Fifteen healthy subjects served as normal controls. Of 15 normal subjects, all had open anterior chamber angle, normal intraocular pressure, no known ocular abnormalities, and a manifest refractive error of -3.00 dioptres or less of myopia, and less than 2.00 dioptres of astigmatism with a best corrected visual acuity of 20/20 or better (11 with normal refractive power, and four with myopia).

\section{Ultrasound biomicroscopy}

Scanning was performed using the Zeiss-Humphrey ultrasound biomicroscope Model 840 (Humphrey-Zeiss, San Leandro, CA, USA). This instrument consisted of a $50 \mathrm{MHz}$ transducer probe, allowing 4-5 $\mathrm{mm}$ tissue penetration and approximately $50 \mu \mathrm{m}$ resolution. The examinations were performed with the patient in the supine position. After surface anaesthesia, eyes were studied using an eyecup, filled with $2 \%$ methylcellulose as a coupling medium. UBM was performed to scan over the central cornea and central anterior chamber, then in $8-12$ sectors of the limbal circumference. The anterior chamber depth was calculated from the distance between the corneal endothelium and the anterior lens surface. All UBM examinations were carried out by a single examiner.

\section{Statistical analysis}

The statistical analysis was processed by SPSS 10.0 software (SPSS Inc, Chicago, IL, USA). Data were expressed as mean (SD). Independent samples $t$ test and $\chi^{2}$ test were used to compare the anterior chamber depth between ICE patients and normal controls, as well as age and sex. A one way
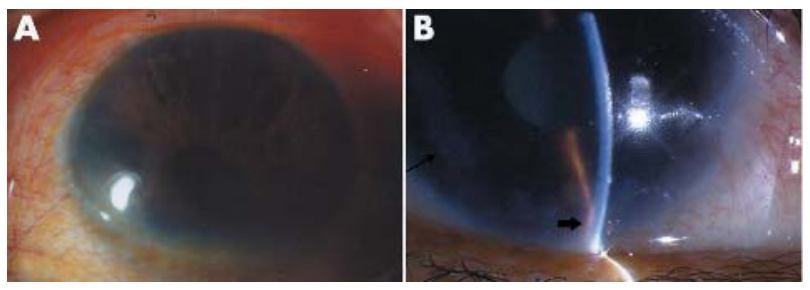

Figure 1 Slit lamp microscopic pictures of ICE patients. (A) Shows iris atrophy, iris holes, and corectopia of a patient with progressive iris atrophy syndrome (patient 1). (B) Shows corneal oedema, iris atrophy (thin arrow), and peripheral anterior synechia (thick arrow) of a patient with Chandler's syndrome (patient 11). analysis of variance (ANOVA) test or $\chi^{2}$ or Fisher's exact test was performed to compare the measurements among the three clinical variants. Statistical significance was considered significant if $\mathrm{p}<0.05$.

\section{RESULTS}

\section{Clinical data}

Patient profiles

Among 21 patients (22 eyes) with ICE syndrome, one patient was found to have CS in the right eye and PIA in the left eye. The remaining 20 presented with unilateral disease. There were 10 men and 11 women. The average age at the time of diagnosis was 49 (SD 13) years (range 33-80 years). For the normal controls, the mean age was 43 (16) years (range 1770 years). There were nine males and six females. The age and sex of normal subjects were similar to patients with ICE syndrome (age: $t=1.344, \quad \mathrm{p}=0.189 ; \quad$ sex: $\chi^{2}=0.161$, $\mathrm{p}=0.688)$. Figure 1 showed slit lamp examination findings of patients with PIA (fig lA) or CS (fig 1B).

\section{Eyes with ICE syndrome}

Ultrasound biomicroscopy data were obtained in 18 eyes ( 17 patients) with ICE syndrome. The patient profiles are shown in table 1 . Best corrected visual acuity was usually poorer in patients with CS than with PIA or CRS. Intraocular pressure (IOP) was obtained by averaging three repeated measurements using non-contact tonometer. The intraocular pressure (IOP) in eyes with ICE syndrome varied widely, ranging from 7.4-59.1 mm Hg. Nine eyes had an IOP higher than $21 \mathrm{~mm} H g$. All CS patients had severe corneal oedema and the fundus was not accessible. Two PIA patients and three CRS patients showed advanced cupping of the optic nerve (C/ $\mathrm{D} \geqslant 0.6$ ). Peripheral anterior synechiae were observed by slit lamp biomicroscopy in 13/18 eyes and iris atrophy was observed in 12/18 eyes with ICE syndrome. Gonioscopy revealed PAS in all patients with PIA or CRS. Patients with CS were not examined by gonioscopy because of the severe corneal oedema. Three of four PIA patients and all CRS patients received surgery, either a trabeculectomy or an aqueous shunt or in some instance both. All patients with CS received at least once penetrating keratoplasty with or without trabeculectomy.

\section{Contralateral eye of unilateral ICE syndrome}

Contralateral eyes of 11 unilateral ICE patients were examined with UBM. The patient profiles are shown in table 2. The BCVA was 20/30 in two eyes and 20/20 in nine eyes. The average IOP was 15.1 (2.2) $\mathrm{mm} \mathrm{Hg}$ (range 10.218.0). Four patients had shallow anterior chamber, and no 

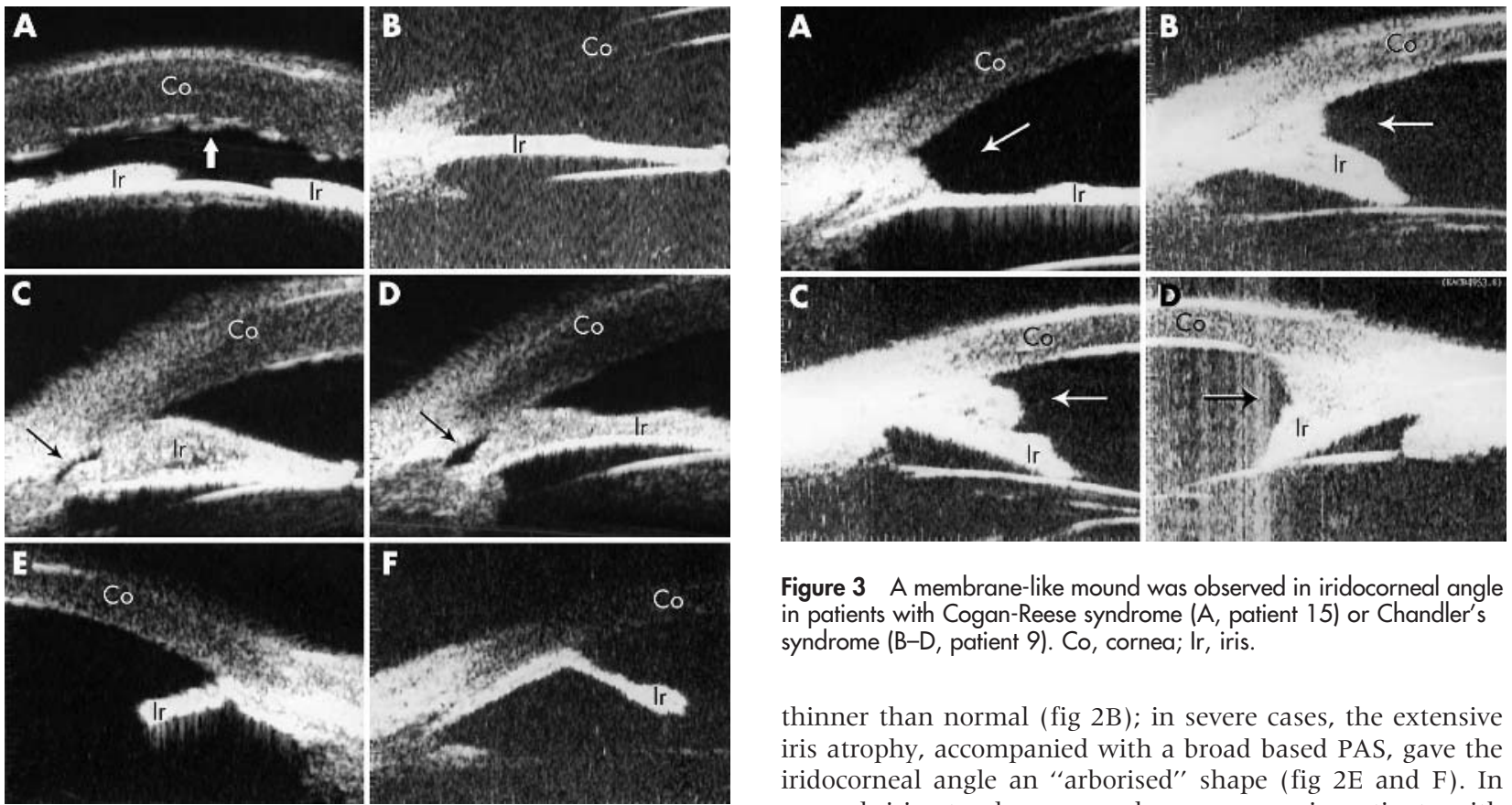

Figure 2 Ultrasound biomicroscopic (UBM) features of ICE syndrome. (A) Shows the shallow anterior chamber and severe corneal oedema in a patient with Chandler's syndrome (patient 5). Folds of Descemet's membrane present a wave-like appearance (indicated by the white thick arrow). (B) Demonstrates the UBM image of a patient with progressive iris atrophy (patient 2). This patient had extensive iris atrophy and peripheral anterior synechiae, which gave rise to severe glaucoma with IOP of $47 \mathrm{~mm} \mathrm{Hg}$ and optic nerve atrophy (cup to disc ratio of 0.9). A "bridge-shaped" peripheral anterior synechia was found in two patients with Chandler's syndrome (patients 10 and 6, respectively), as illustrated in (C) and (D). The iridocorneal angle was indicated by the thin black arrow. Both patients had a shallow anterior chamber with a slightly elevated (patient 10) or normal (patient 6) IOP. (E) and (F) demonstrate the iridocorneal angle with an "arborised" shape caused by severe iris atrophy and broad based peripheral anterior synechiae in two patients with Cogan-Reese syndrome (patients 15 and 16). Both patients had severe glaucoma as judged by IOP elevation and optic nerve cupping. Co, cornea; Ir, iris.

anterior segment abnormality was found in the remaining seven patients. No patient showed advanced cupping of optic nerve.

\section{UBM findings of eyes with ICE}

\section{Corneal oedema}

Corneal oedema was detected in all the patients with CS. The corneal thickness was as great as $1.285 \mathrm{~mm}$ in patient 10 (fig 2C). In some cases, folds in Descemet's membrane occurred, leading to the irregularity in the posterior corneal surface (fig 2A).

\section{Anterior chamber depth}

The central anterior chamber depth (ACD) was significantly less in patients with ICE $(2.25(0.32) \mathrm{mm})$ compared to normal subjects $(2.76(0.32) \mathrm{mm}) \quad(t=3.240, \mathrm{p}=0.003$, independent samples $t$ test). The mean ACD was similar among three clinical variants $(2.41(0.14) \mathrm{mm}$ for PIA, 2.11 (0.59) $\mathrm{mm}$ for CS, and $2.44(0.65) \mathrm{mm}$ for CRS, $F=0.730$, $\mathrm{p}=0.498$, ANOVA).

Iris atrophy

Iris atrophy was observed in all patients except for one patient with Chandler's syndrome. The extent of iris atrophy varied. In mild cases, the iris kept its original shape but was

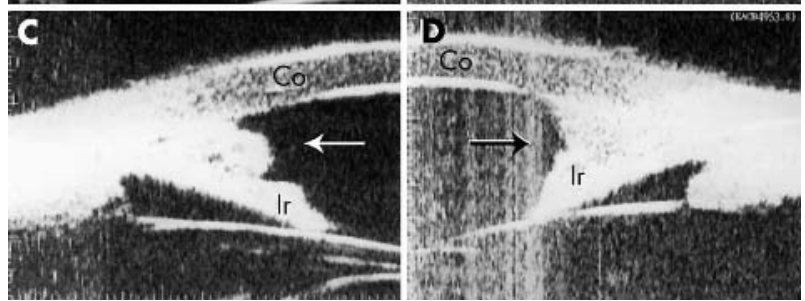

Figure 3 A membrane-like mound was observed in iridocorneal angle in patients with Cogan-Reese syndrome (A, patient 15) or Chandler's syndrome (B-D, patient 9). Co, cornea; Ir, iris.

thinner than normal (fig 2B); in severe cases, the extensive iris atrophy, accompanied with a broad based PAS, gave the iridocorneal angle an "arborised" shape (fig 2E and F). In general, iris atrophy appeared more severe in patients with PIA or CRS than in patients with CS.

\section{Iridocorneal angle}

Peripheral anterior synechiae of different severity were observed in all ICE patients. In patients with CRS, three out of four eyes showed very high PAS, accompanied with extensive iris atrophy, resulting in an "arborised" shape of iridocorneal angle (fig 2E and F). These features were not found in patients with PIA or CS. Two eyes with CS presented a bridge-shaped PAS, in which the middle part of the iris adhered to the cornea, leaving the iridocorneal angle open (fig 2C and D). This was observed only in patients with CS. In two patients, a membrane-like mound was observed in iridocorneal angle (fig 3A-D).

\section{Comparisons among slit lamp biomicroscopy, gonioscopy, and UBM}

Peripheral anterior synechiae were detected by UBM in all 18 eyes with ICE syndrome, whereas only 13 were found to have PAS by slit lamp biomicroscopy. The difference is statistically significant ( $p=0.045$, Fisher's exact test). PAS was found in all patients examined by gonioscopy; however, gonioscopy could not be achieved in CS patients with oedematous
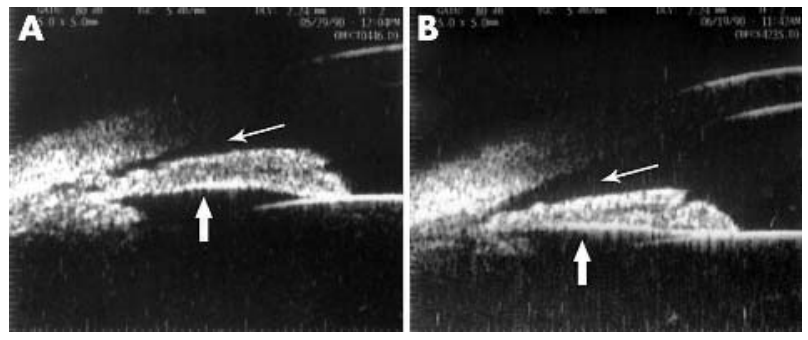

Figure 4 The UBM characteristics of contralateral eye before and after receiving laser peripheral iridectomy. (A) Shows the UBM image of the contralateral eye in a 34 year old female patient with Chandler's syndrome. The fellow eye presented a narrow to closed anterior chamber angle and iris bombé. After laser peripheral iridectomy, the anterior chamber angle was significantly widened (indicated by the thin arrow) and the iris also flattened (indicated by the thick arrow), as shown in (B). Anterior chamber depth was increased from $1.84 \mathrm{~mm}$ to $1.95 \mathrm{~mm}$. 
cornea. Using UBM, 17 eyes with ICE syndrome were revealed to have iris atrophy. The sensitivity was higher than that by slit lamp biomicroscopy (12/18), but the difference was not significant $(\mathrm{p}=0.088$, Fisher's exact test).

\section{UBM findings of the contralateral eyes}

The central anterior chamber depth of the contralateral eyes of ICE patients was $2.35(0.58) \mathrm{mm}$ (range 1.64-3.50 mm). Four out of 11 patients presented shallow anterior chamber depth (average $1.81(0.11) \mathrm{mm}$, range 1.64-1.89) and narrow or closed anterior chamber angle as viewed by UBM (fig 4A). PAS was observed in one of these four patients. Two of them received laser peripheral iridotomy, resulting in the widening of the angle (fig 4B). A ciliary body cyst was found in the contralateral eye of one patient with CRS.

\section{DISCUSSION}

Though uncommon in routine practice, ICE syndrome has attracted much attention both for the enigma in its pathogenesis and the challenge in its diagnosis and treatment once in mind. In cases with atypical clinical features, such as lack of iris hole or corectopia, or with corneal oedema, diagnosis of this rare disorder can be difficult. In the present study, UBM was found to be a good tool in detecting the features of anterior chamber angle region, giving detailed information about peripheral anterior synechiae and iris atrophy, two major clues for diagnosis. It has special merit when the cornea does not permit a good view by slit lamp microscope or gonioscope. In addition, when comparing the UBM findings between PIA, CS, and CRS, distinctive features were found in each of the three variants. In PIA patients, UBM revealed marked iris atrophy and PAS, while the synechiae were not as extensive as those in patients with CRS. In patients with CS, corneal oedema was a common UBM feature. In some severe cases, fold in Descemet's membrane was a further diagnostic feature. Peripheral anterior synechiae were less evident in patients with CS, but a bridge-shaped PAS was presented in two eyes out of ten. Broad based PAS were more pronounced in patients with CRS compared to other two variants of ICE syndrome. Accordingly, an "arborised" PAS, indicating more severe synechia, was often observed in these patients. These features may provide additional criteria to differentiate among the three variants. Gonioscopy has been a common tool to determine the severity of peripheral anterior synechiae before the introduction of UBM. Gonioscopy has proved to be very useful in defining the extent of PAS, whereas UBM provides more information about the contour of the synechiae and the details in the anterior chamber angle region. When combined, these techniques can achieve a more comprehensive understanding of ICE syndrome.

The findings in this study also provide an explanation for some clinical features of ICE syndrome. As observed in previous studies, about half of ICE patients have secondary glaucoma. ${ }^{6-9}$ Among the three clinical variants, Cogan-Reese syndrome and progressive iris atrophy have been suggested to induce more severe glaucoma and a higher failure rate of filtration surgery than does Chandler's syndrome. ${ }^{137}$ This is consistent with our data that the frequency of elevated IOP is much higher in patients with CRS or PIA than in patients with CS. This is also in agreement with our UBM findings that patients with CRS tend to have a higher peripheral anterior synechia. In a previous report, it was noted that the extent of PAS correlates with the reduction of outflow facility and the elevation of IOP. ${ }^{1}$ But in some cases, patients with extensive PAS show minimal IOP alteration; the reason for this is not known. Some authors speculate, yet have not proved, that this might be because the synechiae occasionally bridge the open angle, allowing flow of aqueous behind the synechiae. ${ }^{16}$ By using UBM, we observed bridge-shaped PAS in two CS patients and both patients had normal or slightly increased IOP despite extensive PAS. This finding provides direct support for this speculation. With respect to the deposition observed in the anterior chamber, we are unsure how to explain the mound: it might be another form of PAS, or a membrane covering anterior chamber angle as proposed in the membrane theory of Campbell for the aetiology of ICE syndrome. ${ }^{19}$ However, in a retrospective study, this finding cannot be further explored to find an explanation.

ICE syndrome has been suggested to affect primarily one eye. However, bilateral cases have also been reported..$^{20}{ }^{21}$ In recent years, there is growing evidence about the subclinical abnormalities in the fellow eye. ${ }^{22-24}$ In the present study, a number of ICE patients (4/11) were found to have a narrow angle with normal IOP in the fellow eye. Perhaps it represents an ethnic anatomical difference of anterior angle in an Asian population. Studies have shown that primary angle closure glaucoma (PACG) occurs more frequently in Asians, including Chinese living in mainland China ${ }^{12}$ and Singapore, ${ }^{25}$ people in Thailand ${ }^{26}$ and Mongolia, ${ }^{27}$ than in white populations. The prevalence of PACG in Asians varies from $0.9 \%$ to $1.4 \%$ for the age over 40 , compared to $0.6 \%$ in the same age group in white people. ${ }^{28}$ This may also be because of the average age of ICE patients is over 40, and the PACG prevalence increases while ageing. Therefore, more attention should be paid to the contralateral eyes in ICE patients, especially in the Asian population. In unilateral cases with a narrow angle in the fellow eyes, laser iridotomy may help to protect the contralateral eye from developing PACG.

In summary, UBM, plus gonioscopy, provides a valuable way to diagnose ICE syndrome. Different subtypes of ICE syndrome may have different UBM manifestations. UBM can help to characterise the risk of angle closure glaucoma in the fellow eye of unilateral ICE syndromes.

\section{ACKNOWLEDGEMENTS}

This study was supported by grants from the National Natural Science Foundation of China (2003, 30371514), the National Science Fund for Distinguished Young Scholars of China $(2002,30225044)$, the Jody Sacks Fund to M Zhang and A M Laties, and an unrestricted grant from Research to Prevent Blindness to A M Laties.

\section{Authors' affiliations}

M Zhang, J Chen, L Liang, Z Liv, Zhongshan Ophthalmic Center and Ocular Surface Center, Sun Yat-sen University, Guangzhou 510060, China

M Zhang, A M Laties, Department of Ophthalmology, University of Pennsylvania, Philadelphia, PA 19104-6085, USA

Competing interest statement: No financial interests related to the manuscript.

Ethics approval: Ethics committee approval was not required for this retrospective study.

\section{REFERENCES}

1 Shields MB. Progressive essential iris atrophy, Chandler's syndrome, and the iris nevus (Cogan-Reese) syndrome: a spectrum of disease. Surv Ophthalmol 1979:24:3-20.

2 Alvim PT, Cohen EJ, Rapuano CJ, et al. Penetrating keratoplasty in iridocorneal endothelial syndrome. Cornea 2001;20:134-40.

3 Doe EA, Budenz DL, Gedde SJ, et al. Long-term surgical outcomes of patients with glaucoma secondary to the iridocorneal endothelial syndrome. Ophthalmology 2001;108:1789-95.

4 Kidd M, Hetherington J, Magee S. Surgical results in iridocorneal endothelial syndrome. Arch Ophthalmol 1988;106:199-201.

5 Kim DK, Aslanides IM, Schmidt CM Jr, et al. Long-term outcome of aqueous shunt surgery in ten patients with iridocorneal endothelial syndrome. Ophthalmology 1999;106:1030-4.

6 Shields MB, Campbell DG, Simmons RJ. The essential iris atrophies. Am J Ophthalmol 1978;85:749-59. 
7 Wilson MC, Shields MB. A comparison of the clinical variations of the iridocorneal endothelial syndrome. Arch Ophthalmol 1989;107:1465-8.

8 Hirst LW, Quigley HA, Stark WJ, et al. Specular microscopy of iridocorneal endothelia syndrome. Am J Ophthalmol 1980;89:11-21.

9 Laganowski HC, Kerr Muir MG, Hitchings RA. Glaucoma and the iridocorneal endothelial syndrome. Arch Ophthalmol 1992;110:346-50.

10 Lichter PR. The spectrum of Chandler's syndrome: an often overlooked cause of unilateral glaucoma. Ophthalmology 1978;85:245-51

11 Marchini G, Pagliarusco A, Toscano A, et al. Ultrasound biomicroscopic and conventional ultrasonographic study of ocular dimensions in primary angleclosure glaucoma. Ophthalmology 1998;105:2091-8.

12 Wang N, Wu H, Fan Z. Primary angle closure glaucoma in Chinese and Western populations. Chin Med J (Engl) 2002;115:1706-15.

13 Okamoto F, Nakano S, Okamoto C, et al. Ultrasound biomicroscopic findings in aniridia. Am J Ophthalmol 2004;137:858-62.

14 McWhae JA, Crichton AC, Rinke M. Ultrasound biomicroscopy for the assessment of zonules after ocular trauma. Ophthalmology 2003;110:1340-3.

15 Mannino G, Malagola R, Abdolrahimzadeh S, et al. Ultrasound biomicroscopy of the peripheral retina and the ciliary body in degenerative retinoschisis associated with pars plana cysts. Br J Ophthalmol $2001 ; 85: 976-82$.

16 Gazzard G, Friedman DS, Devereux JG, et al. A prospective ultrasound biomicroscopy evaluation of changes in anterior segment morphology after laser iridotomy in Asian eyes. Ophthalmology 2003;1 10:630-8.

17 Nischal KK, Naor J, Jay V, et al. Clinicopathological correlation of congenital corneal opacification using ultrasound biomicroscopy. Br J Ophthalmol 2002;86:62-9.

18 Madhavan C, Basti S, Naduvilath TJ, et al. Use of ultrasound biomicroscopic evaluation in preoperative planning of penetrating keratoplasty. Cornea 2000;19:17-21.
19 Campbell DG, Shields MB, Smith TR. The corneal endothelium and the spectrum of essential iris atrophy. Am J Ophthalmol 1978;86:317-24.

20 Huna R, Barak A, Melamed S. Bilateral iridocorneal endothelial syndrome presented as Cogan-Reese and Chandler's syndrome. J Glaucoma 1996:5:60-2

21 Blair SD, Seabrooks D, Shields WJ, et al. Bilateral progressive essential iris atrophy and keratoconus with coincident features of posterior polymorphous dystrophy: a case report and proposed pathogenesis. Cornea 1992;11:255-61.

22 Lucas-Glass TC, Baratz KH, Nelson LR, et al. The contralateral corneal endothelium in the iridocorneal endothelial syndrome. Arch Ophthalmol 1997; 115:40-4.

23 Liu Z, Zhang M, Chen J, et al. The contralateral eye in patients with unilateral iridocorneal endothelial syndrome. Zhonghua Yan Ke Za Zhi 2002;38:16-20.

24 Eagle RC Jr, Shields JA. Iridocorneal endothelial syndrome with contralateral guttate endothelial dystrophy. A light and electron microscopic study. Ophthalmology 1987:94:862-70.

25 Foster PJ, Oen FT, Machin D, et al. The prevalence of glaucoma in Chinese residents of Singapore: a cross-sectional population survey of the Tanjong Pagar district. Arch Ophthalmol 2000;1 18:1 105-11.

26 Bourne RR, Sukudom P, Foster PJ, et al. Prevalence of glaucoma in Thailand: a population based survey in Rom Klao District, Bangkok. Br J Ophthalmol 2003;87:1069-74.

27 Foster PJ, Baasanhu J, Alsbirk PH, et al. Glaucoma in Mongolia. A population-based survey in Hovsgol province, northern Mongolia. Arch Ophthalmol 1996;1 14:1235-41.

28 Bonomi L, Marchini G, Marraffa M et al. Epidemiology of angle-closure glaucoma: prevalence, clinical types and association with peripheral anterior chamber depth in the Egna-Neumarkt Glaucoma Study. Ophthalmology 2000;107:998-1003.

\section{1 th European Forum on Quality Improvement in Health Care}

26-28 April 2006, Prague, Czech Republic

For further information please go to: www.quality.bmipg.com

Book early to benefit from a discounted delegate rate 